\title{
CURARE LE ANIME. COSCIENZA DEL PECCATO E FORME DI PENITENZA NELLE REGOLE CENOBITICHE (SECC. V-VII)
}

\section{ROBERTO BELLINI}

\author{
UDC: $27-788 " 04 / 06 "$ \\ 27-42
}

Original scientific paper

Manuscript received: 17. 01. 2017.

Revised manuscript accepted: 01. 02. 2017.

DOI: 10.1484/J.HAM.5.113736

\author{
R. Bellini \\ Università Cattolica del Sacro Cuore \\ Largo Gemelli, 120123 Milano \\ Italia \\ roberto.bellini@unicatt.it
}

\begin{abstract}
Although monastic rules are neither a penal code nor a treatise of moral theology, they provide some reflections on the nature of sin, whose origins are ascribed to the demon's action, to the weakness of the flesh and to secular temptation. This is particularly evident in the Regula Magistri and in saint Columbanus's Regula monachorum. To atone for sins, monastic rules suggest specific penances, the most important of which is excommunication. This includes or, alternatively, considers other forms of mortification, especially corporal punishment and fasting. The closer and closer relationship between a sin and its specific penance finds its culmination in saint Columbanus's regula cenobialis and penitential, which will spread the Irish system of tariff penance on the continent, extending it to the secular clergy and the laity, as well.
\end{abstract}

Keywords: Monastic rules, sin, demon, penance, corporal punishment, fasting, Irish system tariff penance, excommunication, penitential books

Poenitentia vera est paenitenda non admittere, sed admissa deflere: pur essendo stato allievo per molti anni a Bangor dell'abate Comgall, famoso per il suo rigore, ed essendo anch'egli ammirato per il suo stile di vita ascetico, il santo abate Colombano rivelava, in queste espressioni del suo penitenziale, una profonda consapevolezza della fragilità dell'uomo davanti al peccato, anche da parte di chi decideva di abbracciare la non facile strada della vita cenobitica ${ }^{1}$.

Ma quali sono le radici di questa fragilitas? Come si manifesta concretamente il male nel monastero e quali i modi per riscattarlo? Sono domande cui le regole non mancano di rispondere, sia pure non direttamente. Una regola, in effetti, ha lo scopo prioritario di stabilire un ordine e un'armonia alle diverse attività che i monaci conducono nel monastero, traendo i suoi principi dalle Scritture e dalla tradizione, dai modelli offerti dalle grandi figure eremitiche e cenobitiche - esemplare in tale senso è la Vita di Antonio composta da Atanasio - e dall'esperienza pratica condotta da costoro e dai loro discepoli ${ }^{2}$. È soprattutto quest'ultima, mi pare, a giustificare la presenza in esse di numerose prescrizioni particolari di vita e, quindi, a dare loro un carattere non soltanto formativo - espressione di una spiritualità facente capo ad alcuni valori fondamentali, da tradurre in atto nell'esperienza quotidiana sia individuale, sia collettiva - ma anche normativo, benché questo aspetto, che conoscerà un punto di svolta con gli scritti del Maestro e di Benedetto, assumerà il suo significato specificatamente giuridico soltanto molto più tardi³. Alla luce di ciò, se è vero che una regola non è né un trattato di teologia morale, né un codice penale, è tuttavia possibile trovare, in codesti scritti, non poche indicazioni

\footnotetext{
${ }^{1}$ Sigle e abbreviazioni: CI = J.-C. GUY (ed.), Jean Cassien. Institutions cénobitiques, Paris 1965 (Sch, 109); CSEL= Corpus scriptorum ecclesiasticorum latinorum; MGH = Monumenta Germaniae historica; PC = Poenitentiale s. Columbani, in L. BIELER (ed.), The irish penitentials, Dublin 1963 (Scriptores latini Hiberniae, 5), pp. 96-106; PL = J.-P. MIGNE (ed.), Patrologiae latinae cursus completus; R4P, RMac, Ror, 3 RP = Regula quattuor Patrum, Regula Macarii, Regula orientalis, Tertia regula Patrum in A. DE VOGÜÉ (ed.), Les règles des saints Pères, 2 voll., Paris 1982 (Sch, 297-298); RB = A. DE VOGÜÉ - J. NEUFVILLE (edd.), La règle de saint Benoît, 2 voll., Paris 1972 (Sch, 181-182); RBa = K. ZELZER (ed.), Basili regula a Rufino latine versa, Vindobonae 1986 (CSEL, 86); RCae = Sancti Caesarii episcopi regula monachorum, in G. MORIN (ed.), Sancti Caesarii Arelatensi opera omnia, II: Opera varia, Maretioli 1942, pp. 149-155; RCe = Regula cenobialis, in G. S. M. WALKER (ed.), Sancti Columbani opera, Oxford 1957 (Scriptores latini Hiberniae, 2), pp. 142-168; RComm = Regula monastica communis, PL 87, coll. 1111-1127; RComp = Sancti Fructuosi regula Complutensis, PL 87, coll. 1099-1110; RcP = F. VILLEGAS, La "Regula cuiusdam Patris ad monachos". Ses sources littéraires et ses rapports avec la "Regula monachorum" de Colomban, in Revue d'histoire de la spiritualité, 49 (1973), pp. 10-35; RFe = V. DESPREZ, La Regula Ferrioli. Texte critique, in Revue Mabillon, 6o (1981-84), pp. 124-148; RIs = Regula monachorum Isidorii, PL 83, coll. 867-894; RMo = Regula monachorum, in G. S. M. WALKER, op. cit., pp. 122-142; RMag = A. DE VOGÜÉ (ed.), La règle du Maître, 2 voll., Paris 1964 (Sch, 105-106); RPPr, RPIn, RPIu = Regula Pachomii. Praecepta, Instituta, Iudicia in A. BOON (ed.), Pachomiana latina, Leuven 1932 (Bibliothèque de la Revue d'histoire ecclésiastique, 7); RPS = E. M. VILANOVA, Regula Pauli et Stephani. Edicío crítica i comentari, Montserrat 1959 (Scripta et documenta, 11); $\mathrm{RTa}=$ F. VILLEGAS, La «Regula monasterii Tarnantensis». Texte, sources et datation, in Revue benedictine, 84 (1974), pp. 14-46; Sch = Sources chrétiennes. PC A c. 1, p. 96; che Colombano ritenesse, in effetti, più realistica la seconda situazione lo mostra quanto subito segue: quia hanc multorum fragilitas, ut non dicam omnium, rumpit. Sull'austerità dell'ambiente di Bangor cfr. J. B. STEVENSON, The monastic rules of Columbanus, in M. LAPIDGE (cur.), Columbanus: studies on the latin writings, Woodbridge 1997 (Studies in celtic history, 17), pp. 204-205.

${ }^{2}$ È stato soprattutto Isidoro di Siviglia a richiamare esplicitamente, nelle sue opere, questi fondamenti del cenobitismo occidentale, cfr. J. FONTAINE, $L a$ vocation monastique selon saint Isidore de Sévilla, in Théologie de la vie monastique. Études sur la tradition patristique, Paris 1961 (Théologie, 49), pp. 360-361. 3 Sono stati proprio il Maestro e Benedetto a impiegare per primi il termine regola per indicare questa tipologia di fonti: v. A. DE VOGÜÉ, Les règles monastiques anciennes (400-700), Turnhout 1985 (Typologie des sources du Moyen Âge occidental, 46), pp. 11-12. Su tali aspetti delle regole monastiche cfr. la chiara sintesi di M. P. ALBERZONI, La vita religiosa e la sua regolamentazione fino a Francesco, ora in EAD., Santa povertà e beata semplicità. Francesco d'Assisi e la Chiesa romana, Milano 2015 (Ordines. Studi su istituzioni e società nel medioevo europeo, 1), pp. 26-31, con ampi riferimenti bibliografici.
} 
puntuali riguardanti specifiche pene da applicare ai monaci caduti nel peccato nonché considerazioni di più ampio respiro attorno alla natura e all'origine di quest'ultimo, frutto, in entrambi i casi, non principalmente di una riflessione teorica, quanto appunto di sperimentazioni concrete, le quali, tra l'altro, hanno spesso comportato sviluppi ed evoluzioni non solamente da una regola all'altra ma all'interno di un medesimo scritto ${ }^{4}$.

\section{IL PECCATO}

Se prendiamo in considerazione la classificazione cronologica delle regole monastiche antiche, magistralmente proposta da Adalbert de Vogüés, si può rilevare come - nonostante fosse possibile leggere qualche indicazione in proposito nella regola di Basilio e, più ampiamente, nelle Istituzioni cenobitiche di Cassiano - tale argomento sia del tutto assente nelle prime tre regulae sanctorum Patrum dell'area lerinense, atteggiamento del resto proprio pure degli ulteriori due scritti a essa riconducibili. Questi ultimi appartengono alla stessa 'generazione', per dirla col de Vogüé, della RMag, con la quale però la prospettiva muta radicalmente. Una delle sue peculiarità, infatti, è l'acutissimo senso del peccato che la pervade e perciò la continua insistenza sul tema della lotta contro la tentazione ${ }^{6}$. La sua origine è individuata nel libero arbitrio dell'individuo, che ha causato la caduta dell'uomo dal paradiso sulla terra e da lì, inesorabilmente, verso l'inferno se non fossero intervenuti la Croce di Cristo e il battesimo, sicché redeat in paradisum generatio nostra per gratiam, unde cum libero arbitrio ceciderat per offensam ${ }^{7}$. La salvezza, tuttavia, è possibile ma non garantita, appunto perché la volontà individuale, espressione della carne, può ancora volgersi al male: è codesto un tema presente in Cassiano ${ }^{8}$, inquadrato però dal Maestro in una più ampia prospettiva antropologica di tipo tricotomico. L'uomo possiede, infatti, un corpo ex limo terrae, il quale quasi quaedam domus est animae: la sede di quest'ultima è il cuore, che è come una radice da cui si dipartono due rami plus fragiles in peccato, ossia gli occhi e la bocca. Attraverso i primi l'anima scruta ciò che può essere oggetto del suo desiderio, mediante la seconda fa invece fuoriuscire quanto concepisce. Pertanto, onde evitare la caduta, l'anima da un lato claudet concupiscentiis suis oculorum fenestras, volgendoli a terra per non vedere il male, dall'altro, habet [...] anima nostra constitutam portam oris et seram dentium, quam pravo claudat eloquio, facendolo così tornare nel cuore dove pereat in avortione ${ }^{9}$. Tutto ciò pone al centro della vita monastica l'obbedienza, la taciturnità, l'umiltà e la rinuncia ai beni personali al fine di custodire il pensiero, la vista e la parola, ma questo sarebbe impossibile senza la presenza nell'uomo dello spirito: eligit enim spiritus, ut voluntas in nobis Domini fiat, ut iam non perficiat anima quidquid cum prava carne concupiscens sibi suaserat, secondo l'insegnamento della stessa Preghiera del Signore, non a caso commentata dalla RMag nel Thema d'esordio. La conclusione è perciò inevitabile: videte ergo quia quidquid a voluntate nostra eligimus iniuste agnoscitur, et quidquid nolentibus nobis a iubente imponitur iuste proficit rationi $i^{10}$

L'aiuto dello spirito è, del resto, indispensabile poiché, accanto alla carne, l'altra radice del peccato è il diavolo. Circa questa terribile minaccia già Cassiano aveva messo in guardia il monaco, illustrando come Satana si serva dei pensieri, degli umori del corpo e delle illusioni prodotte nel sonno per corrompere la purezza guadagnata con la preghiera comune e personale, spingendo inoltre soprattutto il giovane inesperto a celare eventuali colpe al senior al fine di non esserne corretto ${ }^{11}$. Il Maestro fa suoi tali concetti unendovi, però, un'osservazione: il diavolo può agire solo se gli è offerta l'occasione, se velut nosmetipsos ipsi nos captivantes, hostium nostrum incipiamus magis desiderare quam fugere ${ }^{12}$. Occorre dunque respingere le sue seduzioni, presenti anche in iniziative in se stesse lodevoli ${ }^{13}$, sia praticando le virtù, sia

${ }^{4}$ In proposito V. DESPREZ, Introduction generale, in ID. (cur.), Règles monastiques d'Occident (IVe-VIe siècle). D’Augustin à Ferreol, Bégrolles-en-Mauges 1980 (Vie monastique, 9), pp. 22 e 27-28.

${ }^{5}$ A. DE VOGÜÉ, op. cit. (n. 3), pp. 13-16, con tavola riepilogativa alla p. 14.

${ }^{6}$ Lo ha chiaramente sottolineato A. DE VOGÜÉ, Introduction, in RMag, I, pp. 89-9o.

7 RMag Thp 4-6, I, p. 302. L'interpretazione della vita monastica come 'ritorno al paradiso', a una condizione cioè prossima a quella di Adamo antecedente al peccato originale, è un tema classico nella tradizione cenobitica altomedievale: cfr. J. LECLERCQ, Le monachisme du haut Moyen Âge (VIII ${ }^{e}-X^{e}$ siècles), in op. cit. (n. 2), pp. 441-444 (l'indagine del grande studioso benedettino, in realtà, prende le mosse dal secolo VI).

${ }^{8}$ Ad esempio in CI 1.11.2, p. 52: trattando della veste monastica Cassiano si sofferma sulla cintura che, cingendo i lombi del monaco ed essendo fatta di pelle morta, significat eum [il monaco] mortificationem circumferre membrorum, in quibus libidinis atque luxuriae seminaria continentur.

9 RMag c. 8.5-23, I, pp. 398-402, ma anche c. 11.59-6o, II, p. 20, ove il Maestro fa presente che la parola, una volta uscita dalla bocca, non può più tornarvi: la stretta relazione tra anima e corpo è poi nuovamente richiamata nel c. 69.12-27, II, pp. 298-300. La primaria importanza della dimensione interiore nella lotta contro il peccato è, infine, messa in rilievo mediante una serie di metafore in RMag c. 15.1-10, II, pp. 62-64.

10 Su tutto questo RMag Thp 28 et 4o, I, pp. 306 e 308, ma cfr. anche il c. 1.8o-81, I, p. 346 . Riguardo all'impiego del Pater in ambito monastico mi permetto di rinviare a R. BELLINI, «Dacci oggi il nostro pane quotidiano». Allegoria e realismo negli scritti dei Padri della Chiesa, in G. ARCHETTI (cur.), La civiltà del pane. Storia, tecniche e simboli dal Mediterraneo all'Atlantico, Atti del Convegno internazionale di studio (Brescia, 1-6 dicembre 2014), Spoleto 2015 (Centro studi longobardi. Ricerche, 1), pp. 1580-1584, mentre per il tema dell'obbedienza nel Maestro v. A. DE VOGÜÉ, La communauté et l'abbé dans la règle de s. Benoît, Bruges 1961, pp. 266-268.

${ }^{n}$ CI 2.10.3, p. 76, 3.5.1-2, p. 106, et 4.9, p. 132. Per la RMag Thp 69-70, I, p. 314, e c. 11.2-3, II, pp. 6-8: su di essa A. DE VOGÜÉ, op. cit. (n. 6), pp. 89-9o. Un inquadramento generale attorno alla concezione del diavolo in Occidente durante l'epoca tardo-antica e altomedievale si legge in F. VANDENBROUCKE, s.v., Démon: en Occident, in Dictionnaire de spiritualité, III, Paris 1957, coll. 212-219, da integrarsi per Cassiano con A. GUILLAUMONT - C. GUILLAUMONT, s.v., Démon: littérature monastique, ibid., coll. 208-210. Interessanti indicazioni sulla presenza del demonio nella psicologia monastica altomedievale in G. CREMASCOLI, Corpus diaboli. Sulla demonologia di Gregorio Magno, in Il diavolo nel medioevo, Atti del XLIX Convegno storico internazionale (Todi, 14-17 ottobre 2012), Spoleto 2013 (Accademia Tudertina - Atti dei Convegni del Centro italiano di studi sul basso medioevo, n.s., 26), pp. 66-72; cfr. anche S. BOYNTON, «The devil made me do it»: demonic intervention in the medieval monastic liturgy, in M. RUBIN (cur.), European religious cultures. Essays offered to Christopher Brooke on the occasion of his 8oth birthday, London 2008, pp. 89-106.

${ }^{12}$ RMag Thp 73-74 et 78, I, pp. 314 e 316, proveniente almeno concettualmente da RBa int. 195 et 202, pp. 213-214 e 219-220.

${ }_{13}^{13}$ È il caso contemplato da RMag c. 74.3-4, II, p. 312, di un monaco che, senza permesso del superiore o oltrepassando quanto previsto dalla regola, pratica un digiuno in periodo non consono o troppo prolungato: anche in questo caso, infatti, costui rivendica implicitamente il primato della sua volontà rispetto alla virtù dell'obbedienza, perciò etiam per bonum ei subripit diabolus. Senza collegamento con il diavolo, identica posizione in RB c. 49.8-9, II, p. 6o6. 
affidandosi con fiducia alla guida del superiore e al sostegno della preghiera comunitaria, sia infine dedicandosi con scrupolo al lavoro manuale e intellettuale, mediante il quale è adeguatamente occupato il tempo tra un ufficio e l'altro ${ }^{14}$. Quest'ampia e articolata riflessione giustifica appieno la ben nota immagine del monastero proposta dalla RMag: una scuola dove si impara a servire Dio, seguendo e mettendo in pratica i suoi comandamenti e lottando così vittoriosamente contro le tentazioni, in vista del giudizio escatologico ${ }^{15}$.

È singolare constatare come l'antropologia proposta dal Maestro, fondata su una serie di noti passi paolini e che trova singolari corrispondenze concettuali nell'Adversus haereses di Ireneo di Lione ${ }^{16}$, sia rimasta un unicum tra le regole cenobitiche. Non appare neppure nella RB, a lei strettamente collegata, che pure vede l'origine del male nella volontà disobbediente perché 'oziosa' - cioè debole - e pertanto indica il suo primo rimedio nell'obbedienza, intesa quale sostituzione del proprio volere con quello divino. E questo è tanto più necessario a causa dell'azione del demonio ma, in proposito, la RB si limita a qualche breve cenno, perlopiù tratto proprio dalla sua fonte ${ }^{17}$. Tra le altre regole, solamente quelle di Cesario e di Colombano offrono un'analisi del peccato caratterizzata da una certa unità interna, benché più sintetica rispetto alla RMag. Il presule di Arles, il cui scritto è sostanzialmente coevo a quello del Maestro, trattando della lettura durante la messa ricorda come l'anima, privata della Parola, sia simile alla terra senza l'acqua e al corpo senza cibo. In essa, infatti, è presente un nemico, contro cui è necessario un «combattimento spirituale» affinché non la colpisca «con le sue frecce» facendola perire. Si tratta, ovviamente, del diavolo, il quale si manifesta nei molteplici vizi che possono affliggere il monaco: quanta vitia habueritis, tantos habebitis adversarios. Tuttavia la grazia di Dio ha chiamato il cenobita «dalla tenebrosa intimità di questo secolo al porto della quiete» monastica e lo ha dotato delle armi spirituali atte a combattere purché perseveri nella sua scelta. La prima di codeste è appunto vestis [...] saeculares deponere et religiosas adsumere, seguita dal compiere le buone opere indicate dalla regola per tenere lontane le dulces voluptates seculi huius, annientando altresì ogni vizio con la virtù opposta in una sorta di sana competizione tra confratelli, dalla quale tutti trarranno i più alti benefici ${ }^{18}$. Ė interessante notare come pure in altre due regole dell'area arelatense la radice della colpa sia individuata nel secolo. Nella RFe il diavolo agisce attraverso esso a danno dei monaci, ad esempio grazie alla seduzione esercitata dalle donne ${ }^{19}$ oppure sfruttando passioni quali l'invidia e la cupidigia, definite la prima maximum vitium e da combattere con lo «scudo della carità», la seconda radix omnium malorum e laqueum diaboli, perché generatrice di desideri nocivi e inutili ${ }^{20}$. In altri casi, invece, la mondanità fa tutt'uno con l'amore per la carne, così radicato da consigliare un'attesa di almeno sei mesi prima di ammettere il postulante nel monastero onde si liberi della violenza cui la vita secolare l'ha abituato ${ }^{21}$. Contro tale voluttà si lotta attraverso la preghiera notturna e costumi adeguati, ad esempio indossando vesti semplici e comunque non di lino se poste sul corpo nudo, poiché carnem, quae semper vitio naturae superbit, mollibus foveat [...] et ei - i vestiti - quasi minus valide blandiatur [...] in expugnatione animae ${ }^{22}$. Nella RTa, invece, il demonio non è di frequente ricordato, però anche

${ }^{14}$ Rispettivamente RMag c. 15.14-27 et c. 50, II, pp. 64-66 e 222-238: anche Cassiano aveva sottolineato questa funzione del lavoro: CI 13.1-2 et 14, pp. 82 e 84. La dimensione comunitaria in cui si svolge la lotta contro il male è richiamata ulteriormente da RMag c. 15.45-47, II, p. 70, che coinvolge tutto il gruppo dei monaci di cui il reo di cattivi pensieri fa parte nella sua penitenza.

${ }_{15}$ Tra i molti passi dedicati dal Maestro al tema cfr. per es. RMag Pr. 288-294 et Ths 39-46, I, pp. 324-326: A. DE VOGÜÉ, La règle de saint Benoît, IV. Commentaire historique et critique, Paris 1971 (Sch, 184), pp. 62-65, ha ben lumeggiato il fondamento cristologico di questa concezione del monastero.

${ }^{16}$ Concettuali e non letterali, infatti la RMag non è ricordata nell'informato contributo di M. L. ARDUINI, Alla ricerca di un Ireneo medievale, in Studi medievali, s. III, 21 (1980), pp. 269-299. Per la posizione di Ireneo cfr. A. ROUSSEAU - L. DOUTRELEAU - CH. MERCIER (ed.), Irènèe de Lyon. Contre les hérésies 5.6.1-7.2 et 5.9.1-2, V/2, Paris 1969 (Sch, 153), pp. 72-92 e 106-112: il presule lionese si muove lungo una prospettiva paolina - come appunto la RMag, v. A. DE VOGÜÉ, op. cit. (n. 6), pp. 87-88 - che permette di valorizzare la corporeità alla luce della resurrezione di Cristo in funzione antignostica, diversamente dal successivo orientamento più platonizzante di Origene, il quale comporta il concetto di doppia creazione dell'uomo e un'evidente contrapposizione tra corpo e spirito per l'orientamento dell'anima, cfr. su ciò A. ROUSSEAU, Introduction e Notes justificatives in EAD. - L. DOUTRELEAU - CH. MERCIER, op. cit., V/1, Paris 1969 (Sch, 152), rispettivamente pp. 172-174 e 231-232; A. ORBE, Antropología de san Ireneo, Madrid 1969 (Biblioteca de autores cristianos), pp. 16-31; G. DE SIMONE, Note di antropologia ireneana (A. h. 6.1), in E. CATTANEO - L. LONGOBARDO (cur.), Consonantia salutis. Studi su Ireneo di Lione, Trapani 2005 (Oi christianoi, 1), pp. 191-198; in particolare su Origene anche H. CROUZEL, Origène, précurseur du monachisme, in op. cit. (n. 2), pp. $26-27$. ${ }_{17} \mathrm{Cfr}$. RB Pr. 28, I, p. 418, che trascrive alla lettera RMag Ths 24, I, p. 322; RB c. 53.4-5, II, p. 612, dove raccomanda la preghiera prima dello scambio del saluto con l'ospite propter inlusiones diabolicas, con evidente riferimento a RMag c. 71.5-7, II, p. 304; RB c. 58.27-28, II, p. 632, un caso di abbandono del monastero suadenti diabolo.

${ }^{18}$ RCae cc. 9, 19 et 26, pp. 150, 152 e 154-155: la competizione nelle virtù tra i monaci è uno strumento pedagogico frequentemente proposto dalle regole, nel contesto di un'esigenza educativa che le accomuna tutte, come ha sottolineato A. DE VOGÜÉ, op. cit. (n. 6), pp. 92-93; per un confronto anche con l'educazione degli oblati, si veda G. ARCHETTI, «Sub virga magistri». Custodia e disciplina nell'educazione carolingia dei pueri oblati, in Studi medievali, s. III, 57, 2 (2016), pp. 570-571.

${ }^{19}$ RFe c. 4.1-4, p. 128: a loro va perciò vietato l'accesso al monastero etiam religiosis, perché non potest habitatio in longum esse secura inimico proximo. Segnalo che nella spagnola RComm c. 15, col. ${ }_{1123}$ B, c’è un'ampia trattazione del tema, anche se qui si tratta di monache, relazionandosi con le quali il cenobita fur nocturnus in pectore nostro Christum occidere festinat, come esemplificato dalle vicende di Adamo, di Davide e di Salomone o, se pure fossero parenti, di Thamar e Ammon (per quest'ultimi cfr. RComm c. 17, col. 1124D). Si tratta, nello specifico, di problematiche proprie dell'ambiente claustrale del quale questa regola è riflesso, caratterizzato dalla presenza di monasteri doppi e chiostri 'familiari'’ v. su ciò M. DÍAZ Y DÍAZ, s.v., Fruttuoso di Braga, in Dizionario degli Istituti di Perfezione, IV, Roma 1977, coll. 984-985; ID., Scrittori della penisola iberica, in A. DI BERARDINO (cur.), Patrologia. IV: I Padri latini dal concilio di Calcedonia (451) a Beda, Genova 1996, p. 104.

${ }^{20}$ RFe c. 9 et 14, pp. 130 e 132-133: il secondo caso è esemplificato in RFe c. 20.1-2, p. 134, nella figura dei vagis sive fugitivis monachis che, volendo essere mundi vetus contemptor, in realtà a via salutis distortus exorbitet.

${ }^{21}$ RFe c. 5.2, p. 128: pur essendo il periodo di attesa per il postulante una misura prevista da tutte le regole, Ferreolo mi sembra sia l'unico a giustificarla in tale modo. Simile problematica, come le altre che seguono, adombrano, a mio parere, l'importante tema monastico altomedievale della vocazione cenobitica come 'esilio', messo a fuoco da J. LECLERCQ, op. cit. (n. 7), pp. 438-439.

${ }^{22}$ RFe c. 13.3 et 31.1-4, pp. 132 e 140. Il primo caso si legge anche nella spagnola RComp c. 17, col. 1170D, unico breve accenno al nostro tema: qui l'azione del diavolo è collegata specialmente alle ore notturne, nel corso delle quali perciò pecularibus orationibus et sacris vigiliis maxima ex parte ducendum est. Dopo i pionieristici rilievi di M. BLOCH, La società feudale, Torino 1982 (Reprints, 10), p. 90 (l'ed. orig. apparve tra il 1939 e il 1940), che aveva segnalato il 
qui alle origini del peccato è collocato il contraddittorio desiderio del monaco di trattenere il secolo presso di sé. Ecco perché si ordina al postulante, se possedesse un gregge, di venderlo distribuendo il denaro ai poveri, dummodo in eis nihil potestas resideat e si eviti l'infestatio diabolica. Oppure, si raccomanda ai monaci impegnati nei lavori agricoli di cantare i salmi e non le nenie in uso presso i contadini laici, affinché pure in tale situazione de his quae huius saeculi homines agunt, nihil cura sit monachus. $\mathrm{O}$, infine, di ridurre al minimo i rapporti con la famiglia di provenienza giacché essi, in sé comprensibili ma appunto secolari, possono allontanare il monaco dall'esclusiva cura delle cose divine ${ }^{23}$.

Quanto a Colombano, nella RMo non si sofferma su una casistica precisa ${ }^{24}$, ma mette in scena una vera e propria 'cosmogonia morale', secondo la felice espressione del de Vogüézs, all'interno della quale operano, con ruoli e responsabilità diverse, Dio, Satana e l'uomo. Ė chiaro che bona Deus fecit cuncta quae creavit, invece mala vero diabolus superseminavit dolosa calliditate corrompendo l'opera divina, sicché se all'origine bene e male erano nettamente divisi, come la luce e le tenebre, postquam mala per diabolum boni depravatione esse coeperunt. L'azione satanica è dunque la vera causa del peccato, però a essa continua a contrapporsi la misericordia divina, la cui grazia da un lato nuovamente distingue il bene dal male, dall'altro permette all'uomo di scegliere il primo o dannarsi al secondo, come dimostra l'esempio scritturistico di Caino e di Abele. A tali considerazioni previe segue l'individuazione di quanto rimasto integro dalla corruzione diabolica e, in parallelo, del suo opposto: in particolare, quod declinat a bonitate condita et integritate, hoc primum male est, ossia la superbia, cui si contrappone la piae bonitatis humilis existimatio suum creatorem agnoscentis et glorificantis. Codesto atteggiamento consente al monaco di custodire i beni interiori ed esteriori, evitando la vanità e la disperazione mediante la pratica della temperanza e del discernimento ${ }^{26}$. È soprattutto quest'ultimo, per Colombano, la virtù essenziale: discretio igitur discernendo nomen accepit eo quod ipsa in nobis discernit inter bona et mala e se la temperanza ci spinge a macerare la carne quale indispensabile via per un autentico spiritalis profectus, d'altra parte inter parvum et nimium est in medio mensura [...] et haec mensurae verae discretionis, grazie alla quale nequaquam nos deviare $a b$ iusto permittet ${ }^{27}$. Dunque, è forse troppo azzardata l'immagine talvolta proposta di un Colombano esasperato asceta, che considera l'uomo una 'bestia feroce' da ricondurre all'ordine e alla disciplina con l'impiego del bastone e della frusta. Semmai, appare in lui fortemente presente il senso della drammatica scelta che l'uomo deve continuamente compiere, spettando al libero arbitrio, in ultima analisi, decidere del suo destino ${ }^{28}$. E poiché la persona è fragile e la risoluzione quindi difficile, per l'abate irlandese maxima pars regulae monachorum mortificatio est, in altre parole nihil sine consilio faciendo, totum per consilium est interrogandum. Affidarsi alla guida dell'abate o del senior, all'apparenza anche dura e severa ma in sé traboccante d'amore, confidando loro con fiducia debolezze e colpe è, per Colombano, la strada regia verso gli autentici bona Dei e l'essenza stessa della vera vita cenobitica: cavenda ubique est monachis superba libertas ac vera humilitas discenda, sine murmuratione et haesitatione oboedientibus ${ }^{29}$, un principio ripreso, sia pure per cenni, dalla RcP, che indentifica nella stolta pretesa di sapere e di potere guidare se stesso il vizio principale sfruttato dal demonio per condurre il monaco alla rovina ${ }^{30}$.

Per completare questa prima ricognizione, restano da segnalare alcuni passi contenuti nelle RPS e nella RIs, due scritti differenti per cronologia earea geografica, accomunati alla maggior parte delle regole dal non eseguire una meditazione sistematica attorno al nostro tema, di cui toccano

timore con il quale l'uomo medievale scrutava la notte, questo problema ha ricevuto una crescente attenzione da parte della storiografia: si v. J. DELUMEAU, La paura in Occidente (secoli XIV-XVIII). La città assediata, Torino 1979, pp. 136-149; V. FUMAGALLI, L'alba del medioevo, ora in ID., Paesaggi della paura. Vita e natura nel medioevo, Bologna 1994, pp. 37-44; D. YOUNGS - S. HARRIS, Demonizing the night in medieval Europe: a temporal monstruosity?, in B. BILDHAUER - R. MILLS (cur.), The monstrous middle ages, Cardiff 2003, pp. 134-154, e soprattutto le due ampie monografie di J. VERDON, La nuit au Moyen Âge, Paris 1995, che mette in rilievo anche gli aspetti 'sublimi' della notte, intesa quale momento in cui era possibile un maggiore avvicinamento a Dio, e di T. BOIADIJEV, Die Nacht im Mittelalter, Würzburg 2003, con largo spazio dedicato proprio alle inlusiones notturne; qualche puntualizzazione in G. ARCHETTI, op. cit. (n. 18), pp. 559-56o.

${ }^{23}$ RTa cc. 1.10-13, 8.14-16 et 12.9-11, p. 16, 26 e 31 rispettivamente. Nella casistica delle punizioni i rapporti soprattutto epistolari con la famiglia di provenienza sono una costante, fin dalla regola di Pacomio. Si noti altresì che il primo problema, pure esso nella sua generalità un classico della letteratura monastica, è svolto in forma pressoché analoga in RIs c. 4.1, col. $871 \mathrm{C}$, benché sembra si debbano escludere dipendenze dirette tra i due scritti (cfr. F. VILLEGAS, La «Regula monasterii Tarnantensis», cit., pp. 62-65).

${ }^{24}$ Perché questo scritto è espressione soprattutto della sua spiritualità e dunque finalizzato a formare il monaco nelle virtù che sono a lui proprie, laddove all'analisi specifica del peccato e alla determinazione delle indispensabili penitenze atte a redimerlo sono destinate la RCe e il PC: $v$. in proposito le osservazioni di A. DE VOGÜÉ, Aux sources du monachisme colombanien, II. Saint Columban, Règles et pénitentiels monastiques, Bégrolles-en-Mauges 1989 (Vie monastique, 20), pp. 48-49, 51 e 89, e di J. B. STEVENSON, op. cit. (n. 1), pp. 206-207; sulla RMo cfr. anche G. S. M. WALKER, Introduction, in ID. (ed.), op. cit., pp. XLVI-XLVII.

${ }_{25}^{25}$ A. DE VOGÜÉ, op. cit. (n. 24), p. 44.

${ }^{26}$ Quest'ampia riflessione del santo irlandese si legge in RMo c. 8 (10-12), pp. 134-136.

${ }^{27}$ RMo cc. 3 (5), 8 (10) et 13, pp. 126, 134 e 136. Sulla discretio, concetto tra i più importanti della RB ma, come si vede, fatto proprio pure da Colombano, cfr. F. DINGJEAN, Discretio. Les origines patristiques et monastiques de la doctrine sur la prudence chez saint Thomas d'Aquin, Assen 1967, pp. 14-144; per Colombano A. DE VOGÜÉ, op. cit. (n. 24), p. 44; con specifico riferimento a Benedetto E. SCHOLL, The mother of virtues: discretio, in Cistercian studies quarterly, 36 (2001), pp. 389-401; M. M. CEREZO-RÉLLAN, La discretio nella regola di s. Benedetto, in Inter fratres, 63 (2013), pp. 160-170.

${ }^{28}$ Lo ha rilevato A. DE VOGÜÉ, op. cit. (n. 24), pp. 31-32 e 77.

${ }^{29}$ Entrambi i passi si leggono in RMo c. 9 (14), p. 138. Riguardo a questo principio colombaniano, fondamentale dell'intero movimento cenobitico - non a caso è, infatti, ricordato da tutte le regole - A. DE VOGÜË, 'Ne juger de rien par soi-même'. Deux emprunts de la règle colombanienne aux Sentences de Sextus et à saint Jérôme, ora in ID., Regards sur le monachisme des premiers siècles, Roma 2000 (Studia Anselmiana, 130), pp. 445-451, ha messo in chiaro come l'abate irlandese, attraverso la ripresa di codeste due auctoritates, conferisca all'obbedienza un significato sia di tutela verso il monaco da parte del superiore, che si assume la responsabilità davanti a Dio del suo profectus, sia di obbligo rigoroso, da parte del cenobita, di sottomettersi alla volontà della sua guida. ${ }^{30}$ RcP c 1.5, p. 10: proprio il passaggio dall'eremitismo alla vita cenobitica, secondo CH. COURTOIS, L'évolution du monachisme en Gaule de st Martin à st Colomban, in Il monachesimo nell'alto medioevo e la formazione della civiltà occidentale (Spoleto, 8-14 aprile 1956), Spoleto 1957 (Settimane di studio del 
solo aspetti specifici ${ }^{31}$. La RPS vede emergere la tentazione diabolica innanzi tutto nei momenti di riposo, sicché si raccomanda ai monaci impegnati nel lavoro dei campi di non dormire divisi, sed in uno loco aut in duobus si ristorino dalle fatiche nel corso dei pomeriggi estivi. Altri momenti pericolosi sono le attività dell'ufficio divino e le esplosioni di riso immoderato, definite codeste ultime ianuam per quam perniciosos cibos miserae animae diabolos subtiliter subministrat $^{32}$. Nella RIs, oltre a un breve accenno al demonio, la radice della colpa è soprattutto collocata nelle sollecitazioni della carne, pertanto il suo principale rimedio è identificato nella sobrietà, sia a mensa - nam ex plenitudine ventris cito excitatur luxuria carnis - sia nel vestiario, che deve essere non delicatus in entrambi i sensi: infatti, se pretiosa vestis animum ad lasciviam pertrahit, un abito troppo vile morbum vanae gloriae contrahit. Analoghe ragioni devono sconsigliare l'affidare incarichi all'esterno del chiostro a monaci adolescenti o poco formati, ne infirma aetas carnis desiderio polluatur o la severità dell'esperienza cenobitica risvegli in loro le lusinghe della vita secolare ${ }^{33}$.

\section{LA PENITENZA}

Conoscere la natura e le origini del peccato non è, ovviamente, sufficiente per evitarlo, anzi, le regole ritengono scontata la caduta del monaco in questa o quella colpa. La penitenza appare allora come la provvidenziale medicina dell'anima, in grado di risanarla e di permetterle di proseguire il suo itinerario verso la perfezione. Efficacissima è special- mente la RCe di Colombano nel sottolineare come questo sia lo scopo principe delle sanzioni e ciò aldilà dei loro aspetti strettamente disciplinari, che pure non mancano essendo il monastero un sistema di vita comunitario, la cui stessa esistenza è ontologicamente impossibile in assenza di norme e di punizioni proporzionate per chi le avesse violate ${ }^{34}$. Infatti, diversitas culparum diversitatis poenitentiae medicamento sanari debet, dunque la pena non può essere lasciata al caso o alla libera improvvisazione di chi è delegato a somministrarla, ma costui deve operare col dovuto discernimento affinché essa sia realmente efficace ${ }^{35}$. È ben noto come sia stata proprio la peregrinatio dei monaci insulari, dei quali Colombano fu senz'altro uno dei più rappresentativi esponenti, a diffondere nel continente la prassi della penitenza tariffata, veicolata dai libri penitenziali originariamente in uso presso la Chiesa irlandese ${ }^{36}$. Tuttavia, è opportuno ricordare che già le più antiche regole presentano dei capitoli strutturati secondo tale logica: in particolare gli scritti di Pacomio, introdotti in Occidente dalla traduzione di Girolamo, prevedono una ricca tipologia di colpe cui sono associate puntuali penitenze e persino le Istituzioni di Cassiano, che di per sé non sono una regola, dedicano spazio nel libro IV a questo argomento ${ }^{37}$. Immediata fu quindi la ricezione della procedura nei nostri testi, dapprima in misura limitata ma rapidamente sempre più crescente, varia e articolata.

Non è possibile in questa sede, e neppure utile in generale, una dettagliata rassegna sulle colpe specifiche imputate ai cenobiti dalle diverse regole: una sintesi tipologica può essere riassunta nella seguente tabella.

\begin{tabular}{|l|l|}
\hline \multicolumn{1}{|c|}{ occasione } & \multicolumn{1}{c|}{ tipo di peccato } \\
\hline momenti comunitari & ritardo o condotta superficiale a mensa, all'ufficio e durante il riposo \\
\hline attività individuali & condotte inadeguate durante la lettura, il lavoro e fuori dal monastero \\
\hline rapporti interpersonali & $\begin{array}{l}\text { vaniloquio, parole malvage, ira, mancanza di umiltà, furto, prepotenza nell'esercizio dell'autorità, lussuria (soprattutto } \\
\text { con i monaci giovani) }\end{array}$ \\
\hline rapporti con l'autorità & atteggiamento di contesa, disobbedienza, ribellione, mormorazione, mancata emendazione, violazione della scomunica \\
\hline verso se stessi & $\begin{array}{l}\text { cattivi pensieri, polluzioni, ricezione di lettere e regali senza permesso, renitenza nel confessare le colpe, leggerezza nella } \\
\text { condotta e nelle parole }\end{array}$ \\
\hline
\end{tabular}

Centro italiano di studi sull'alto medioevo, 4), pp. 6o-61, ha reso centrale il valore dell'obbedienza, permettendo così un accesso più largo all'esperienza ascetica poiché la perfezione richiesta era minore e vi era la possibilità di un sostegno nel cammino verso essa.

${ }^{31}$ La RPS appartiene, infatti, alla 'generazione' precedente all'altra ed è di origine italica, pur non presentando influenze né dalla RMag, né dalla RB; la RIs, invece, si deve all'illustre penna del vescovo Isidoro di Siviglia ed è alla base delle successive regole di area ispanica: sorprende un poco, in effetti, considerata la statura intellettuale del suo estensore, la mancanza in essa di una riflessione specifica attorno al nostro tema.

${ }^{32}$ Rispettivamente RPS c. 11, p. 112, e cc. 14 et 37, pp. 113-114 e 123: l'ultimo atteggiamento è spesso deplorato dalle regole, cfr. J. LE GOFF, Il riso nelle regole monastiche dell'alto medioevo, ora in ID., I riti, il tempo, il riso. Cinque saggi di storia medievale, Milano 2002, pp. 158-174, e più in generale T. A. KEMPER, «Iesus Christus risus noster». Bemerkungen zur Bewertung des Lachens im Mittelalter, in A. GRAUBE - N. STAUBACH (cur.), Komik und Sacralität. Aspekte einer äesthetischen Paradoxie im MIttelalter und früher Neuzeit, Frankfurt a. M., 2005 (Tradition - Reform - Innovation. Studien zur Modernität des Mittelalters, 9), pp. 16-31; G. ARCHETTI, op. cit. (n. 18), pp. 570-573. Quanto all'ufficio, singolare ne è la trattazione: si vieta, infatti, di cantare responsori e antifone estranee alla tradizione scritturistica affinché i monaci, irretiti da queste nuove melodie, non siano spinti a disprezzarla o, addirittura, ad abbandonare il chiostro.

${ }^{33}$ Rispettivamente RIs cc. 9.4, 12.1 et 23.2, coll. 878CD, 881C-882A e 853A: a proposito di quest'ultima norma J. FONTAINE, op. cit. (n. 2), pp. 360-361, ne ha efficacemente sottolineato l'aspetto di 'rottura col mondo', caratteristico della spiritualità monastica isidoriana per il quale egli è debitore a Gregorio Magno, riletto però in termini principalmente moralistici.

${ }^{34}$ RCe c. 1, p. 144: confessio et paenitentia de morte liberant, un concetto ribadito nel PC B Prol., p. 98, dove si legge che la penitenza riconduce la debolezza dell'anima alla piena salute, come fanno i medici verso i corpi degli uomini. Mette conto osservare che sin dalle prime regole è sottolineata questa finalità curativa della penitenza, cfr. ad es. RBa int. 23, p. 72 - in cui pure si paragona chi punisce a un padre e a un medico, in quanto identico è il suo spirito verso il peccatore - o R4P 5.16.15-18, I, p. 204.

35 La citazione proviene da RCe Prol., p. 144; PC A c. 1, p. 96, sviluppa il principio conseguente: ut iuxta magnitudinem culparum etiam longitudo statuatur poenitentiarum. Anche in questo caso, però, trattasi di tradizione antica, essendo il concetto iuxta mensuram peccati già presente in Pacomio (cfr. ad es. RPIn cc. 9 et 10, pp. 56-57).

${ }^{36}$ Per i diversi aspetti di questa peregrinatio si v. A. ANGENENDT, Die irische Peregrinatio un ihre Auswirkungen auf dem Kontinent vor dem Jahre 8oo, in H. LÖWE (cur.), Die Iren und Europa im frühen Mittelalter, Stuttgart 1982, pp. 52-79, e A. SCHARER, Insular mission to the continent in the early middle ages, in C. LEYSER - H. WILLIAMS (cur.), Mission and monasticism, Roma 2013 (Studia Anselmiana, 158 - Analecta monastica, 13), pp. 55-62. Sul ruolo di Colombano all'interno del fenomeno cfr. T. M. CHARLES-EDWARDS, Early christian Ireland, Cambridge 200o, pp. 344-345; G. ARCHETTI, Da Bangor a Bobbio: il monachesimo tra VI e VII secolo, in ID. (cur.), Teodolinda. I longobardi all'alba dell'Europa, Secondo convegno internazionale del Centro studi longobardi (Monza, Gazzada Schianno, Castelseprio-Torba, Cairate, 2-7 dicembre 2015), Spoleto 2017 (Centro studi longobardi. Convegni 2), in corso di stampa. ${ }^{37}$ Ho potuto sommariamente contare per Pacomio 47 capitoli penitenziali nelle quattro parti in cui si articola la sua regola, mentre per Cassiano, oltre a isolate indicazioni sparse nei vari libri, cfr. soprattutto CI 4.16.1-3, pp. 140-142, di cui è chiara l'ispirazione pacomiana. 
Oltre al famoso passo di Cassiano riguardante i vizi capitali, tra le regole dei secoli V-VI solo nella RMag si legge un duplice elenco di peccati dai quali è necessario guardarsi ${ }^{3}$. Simile quadro riappare, nel secolo VII, nelle ispaniche RIs e RComp di san Fruttuoso, nella prima con accurata divisione tra colpe lievi e gravi, nella seconda con immediato rinvio alla penitenza adatta a emendarle, il che suggerisce senz'altro l'influenza su di essa della prassi irlandese, la quale conobbe proprio in Spagna un'iniziale benché contrastata applicazione ${ }^{39}$. Tra i numerosi peccati un cenno merita almeno la mormorazione, unanimemente denunciata come atteggiamento più di ogni altro estraneo all'autentica esperienza cenobitica, manifestandosi in essa - a prescindere dalla casistica - il rifiuto di sottomettere la volontà individuale a quella del superiore e alle norme della regola, indizio perciò di uno spirito superbo e del tutto privo di umiltà e tale pertanto da mettere in pericolo la stessa sopravvivenza della comunità. Si comprende così perché la mormorazione sia deplorata sin dalle prime regole e non necessariamente sia trattata in rapporto a una specifica casistica, ma esortando i monaci a evitarla in una pluralità di situazioni differenti $4^{\circ}$.

Qualsiasi colpa, comunque, può essere emendata ${ }^{41}$, di qui l'insistenza delle regole affinché il peccatore non abbia timore a confidarsi con il suo senior: già codesta è una forma di umiltà e di umiliazione, valida per ottenere il perdono. E poiché, come si è accennato, obiettivo fondamentale rimane la salvezza dell'anima del reo, i nostri testi invitano costantemente chi avesse saputo di una caduta altrui a riprendere il colpevole in forma riservata e, se poi non intendesse emendarsi, a denunciarlo al superiore. Benché ciò possa apparirci sgradevole, non si deve dimenticare né la dimensione fortemente comunitaria connotante la vita claustrale, alla luce della quale un peccato non può mai essere considerato un evento strettamente personale - non fosse altro per il cattivo esempio che la sua mancata punizione potrebbe offrire ai meno spiritualmente dotati - né il ruolo imprescindibile che in essa svolge il riferimento alla Scrittura, nello specifico il passo di Mt 18, 15-17, non a caso continuamente richiamato dalle regole ${ }^{42}$.

È dunque il superiore il ministro ordinario della penitenza, in primo luogo l'abate, anche laddove non è indicato con tale nome, specialmente per le colpe importanti ${ }^{43}$. Molti reati, però, sono delegati ai suoi collaboratori, le cui figure e titoli vanno precisandosi da una regola all'altra. Nella RMag un ruolo importante lo ricoprono i praepositi, cui è ordinato di intervenire ogni qual volta un monaco loro affidato cada nel vaniloquio perché codesto non assuma poi forme più serie, dunque con una finalità preventiva ${ }^{44}$. Nella RComp, invece, la sede principale deputata a sanzionare i peccati è la collecta, ter per omnem hebdomadam facienda: qui, letta la regola, a seniore et castigatio ac sermo aedificationis proferendo ad fratres, in modo tale che ne fortasse dolositate et malitia senioris innocens iunior opprimatur e ciascuno sia così giudicato secondo giustizia ${ }^{45}$.

Esattamente come per i reati, non è possibile un'analisi dettagliata delle penitenze previste dalle regole, variando esse continuamente pure tra scritti tra loro interdipendenti persino riguardo alla medesima fattispecie. Del resto, un altro principio spesso rammentato è il primato della discretio pure in questo ambito, quindi non c'è alcuna automaticità nell'irrogare la punizione che è, invece, sempre sottoposta all'attenta ponderazione del superiore. In una panoramica generale, osserviamo in primo luogo che tutti i nostri scritti prevedono sempre una serie di richiami per il peccatore prima di colpirlo con la sanzione, essendo codesta inflitta con animo di padre e di medico verso un figlio ammalato ${ }^{46}$.

\footnotetext{
${ }_{38}^{8}$ Rag c. 5.1-11, I, p. 379 - 33 vizi, non tutti specifici dei monaci - e c. 92.11-25, II, pp. 412-414 - 15 peccati, sostanzialmente già presenti, salvo due, nel primo elenco. Per Cassiano CI 5.1, p. 190.

${ }^{39}$ Lo suggerisce un notissimo canone del concilim Toletanum II a. 589 c. 11, PL 84, col. 353CD, che con rammarico constata come per quasdam Hispaniarum ecclesias si sia diffusa l'abitudine di fare penitenza non secundum canonem, ma ut quotiescumque peccare voluerint, toties a presbitero se reconciliari expostulent. Che si tratti della penitenza tariffata lo ha rilevato G. PICASSO, Dolore dei peccati, espiazione e perdono in alcuni libri penitenziali, in Annali di scienze religiose, 3 (1998), pp. 133-134, mentre per la diffusione di essa in Spagna e i successivi sviluppi si v. G. MOTTA, Libri penitenziali e 'cura animarum', in La pastorale della Chiesa in Occidente dall'età ottoniana al concilio Lateranense IV, Atti della XV Settimana internazionale di studio (Mendola, 27-31 agosto 2001), Milano 2004 (Storia. Ricerche), pp. 58-61. I passi delle due regole si leggono rispettivamente in RIs c. 17.1-3, coll. 885B-886C - molti dei suoi peccati sono ripresi da CI 4.16, pp. 140-142 - e RComp cc. 15-16, coll. 116oB-1107B.

${ }^{40}$ Evitando un'inutile e prolissa casistica, mette conto osservare come la gravità del reato trovasse un fondamento nelle stesse lettere paoline, ad es. 1 Cor 10,10; per le regole monastiche cfr. V. DESPREZ, op. cit. (n. 4), p. 50.

${ }^{41}$ Facendo eco alle pagine evangeliche, RBa int. 27, p. 75, invita infatti ad accogliere con gioia il peccatore pentito.

${ }^{42}$ A. DE VOGÜÉ, Les communautés des moines au regard de Nouveau Testament, ora in ID., op. cit. (n. 29), pp. 83-95, ha messo in luce lo strutturarsi della comunità monastica secondo il modello della comunità apostolica, sia pre sia post pasquale; sul rapporto tra monachesimo e Scritture cfr. inoltre J. BIARNE, La Bible dans la vie monastique, in J. FONTAINE - CH. PIETRI (cur.), Le monde latine antique et la Bible, Paris 1985 (Bible de tous les temps, 2), pp. 409-429. ${ }^{43}$ La prima a sottolineare questo ruolo centrale dell'abate, anche rispetto ai suoi collaboratori, è RMac c. 27, I, p. 384-386, posizione subito ripresa da Ror c. 1.7-9, II, p. 462.

${ }^{44}$ RMag c. 11.40-9o, II, pp. 16-26, ma nei passi successivi la sorveglianza del prevosto è estesa a tutti i momenti della vita cenobitica, tanto che l'abate in adsignato sibi aliquo laboris opere [...] a ceteris fratribus remissios exigatur.

${ }^{45}$ RComp cc. 15 et 20, coll. 1160AB e 1109A: secondo J. PEREZ DE URBIEL, s.v., Regula Complutensis, in Dizionario degli Istituti di Perfezione, VII, Roma 1983, coll. 1570-1571, tale orientamento 'democratico' sarebbe un riflesso delle tradizioni politiche visigotiche, tenute presenti da Fruttuoso - del resto uomo di origine aristocratica - nell'organizzazione delle sue fondazioni. Tuttavia, già nella RIs c. 18.4, col. 887B, era previsto che, per i reati non comportanti la scomunica, in collectam abbati vel praepositi deferentur, un principio ripreso dalla RComm - cfr. ad es. c. 13, coll. 1120D-1121D - in forma però meno netta: RComm c. 11, coll. 1119D-1120A, attribuisce infatti all'abate e al preposito l'excommunicandi [...] causam, aggiungendo quod fecerit [il preposito] semper in arbitrio pendeat abbatis. Nel complesso, l'intervento della colletta appare dunque una prassi caratteristica del monachesimo iberico: questa e simili peculiarità sono state tratteggiate da A. MUNDÒ, Il monachesimo nella penisola iberica fino al sec. VII. Questioni ideologiche e letterarie, in op. cit. (n. 30), pp. 97-100 e 103-106.

${ }^{46} \mathrm{Si}$ v. sopra, alla n. 34, il passo della RBa, che aggiunge: et tunc maxime, cum qualitas curae tristior videtur et gravior: un inquadramento complessivo sulla riflessione del grande Padre di Cesarea in materia in A. DE VOGÜÉ, Les Grandes Règles de saint Basil. Un survol, ora in ID., op. cit. (n. 29), pp. 123124,129 e 131-132.
} 
Va per altro aggiunto che codesti ammonimenti spesso sono già in se stessi una prassi penitenziale: nelle regole pacomiane, anzi, l'increpatio, prevalentemente pubblica, è indubbiamente la punizione predominante perché comporta, appunto, l'umiliazione del reo e, se accolta con adeguato atteggiamento di umiltà e accompagnata dalla richiesta di perdono, estingue subito la colpa ${ }^{47}$. Nella RMag e nella RB è impiegata soprattutto per i ritardi all'oratorio o alla mensa ${ }^{48}$, ma nella RFe colpisce i superbi, usi a contraddire i superiori, e nella RTa i monaci sorpresi in atteggiamenti di eccessiva familiarità verso i confratelli, specialmente se giovani, dunque per colpe sicuramente gravi ${ }^{49}$.

Non tutti i cenobiti, tuttavia, davano soddisfazione a codesta sanzione sicché seguivano misure maggiormente energiche, la più importante delle quali era la scomunica. Simile modo di punizione, da non confondersi con quella ecclesiastica ${ }^{50}$, è isolatamente contemplato nelle prime regole, ma già nella $\mathrm{R}_{4} \mathrm{P}$ dà luogo a un breve codice penale, ossia a una procedura strutturata comprendente alcuni precisi passaggi e caratterizzata da contenuti penitenziali puntuali, applicabili in teoria a qualsiasi fattispecie di peccato ${ }^{51}$. A conferma del fatto che questa tipologia di scritti si arricchì progressivamente attraverso l'esperienza, nelle successive regole dell'area lerinense si coglie agevolmente lo svolgersi di tale codice, che trova il suo modello ultimo nella ROr. In essa sono indicati una serie di atti preparatori i quali portano, in caso di resistenza del peccatore, alla scomunica, a sua volta articolata in successive forme di penitenza - dal digiuno alla degradazione, dal silenzio durante l'ufficio alla separazione fisica dai confratelli - per giungere, infine, all'espulsione dal chiostro, onde evitare il diffondersi nella comunità del suo cattivo esempio ${ }^{52}$.
Il perfezionarsi della procedura mette capo alle due grandi regole italiane, la RMag - coeva alla ROr - e la RB, i cui stretti rapporti col Maestro sono stati minutamente lumeggiati dal de Vogüé. Molteplici sono, in effetti, le loro concordanze circa il nostro tema: entrambe fanno precedere la scomunica da tre richiami - di nuovo è operante il fondamento matteano - e soprattutto determinano la penitenza distinguendo le colpe leggere da quelle rilevanti, prevedendo per le prime il ritardo nella refezione, affinché il colpevole mangi da solo, e il suo silenzio nel corso dell'ufficio, durante il quale deve pure umiliarsi pubblicamente, restando in ginocchio secondo il Maestro, prostrandosi a terra in Benedetto ${ }^{53}$. Diversa è, invece, la prassi per i reati gravi, non tanto nei contenuti penitenziali - separazione dall'oratorio, dalla mensa, dal colloquio con i fratres e nel lavoro, e umiliazione pubblica - quanto nello spirito che la anima. Nella RMag, infatti, è articolata una vera e propria cerimonia - esposta in verità con una certa confusione - inaugurata da una lunga e severa ripresa pubblica del reo da parte dell'abate e conclusa, dopo il perdono, dal suo ammonimento a non ricadere ulteriormente nel peccato, non essendo contemplata una nuova possibile espiazione: dunque egli sarà espulso dal monastero, quae cum secundo fuerit acta, in haereticorum incidet sectam. Tale sorte, preceduta da una razione di verberate, sarà comunque a lui riservata se non si emenderà entro tre giorni dall'imposizione della scomunica ${ }^{54}$. Nella RB, invece, oltre a una superiore chiarezza espositiva, prevale sicuramente la preoccupazione di recuperare il colpevole e perciò un'impostazione pastorale e non prioritariamente liturgica, come nella RMag55. Ne sono prova le belle raccomandazioni rivolte all'abate affinché sia molto attento nei confronti dello

\footnotetext{
${ }_{47}$ A titolo di es. cfr. RPPr c. 8, p. 15 - parlare e ridere durante la preghiera - ove si precisa che il reo, nel ricevere il rimprovero, solvet cingulum [...] inclinata cervice [...] stabit ante altare, mentre al c. 38, p. 25 - trascurare un ammalato - si puntualizza: increpatione solita emendabitur, dunque senza ulteriore pena.

${ }^{48} \mathrm{Cfr}$. ad es. RMag c. 73.1-18, II, pp. 306-310, secondo una casistica articolata e in combinazione con altre pene se il ritardo risultasse importante, e RB c. 43.13-17, II, p. 590, con punizione aggravata se ripetuto. Benedetto impiega tale misura anche per altri peccati, ad es. per chi non custodisce con cura le cose del monastero (RB c. 32.1-5, II, p. 560).

${ }_{49}$ Rispettivamente RFe c. 39.12-15, p. 146, che prevede anche la degradazione, una sanzione non presente nell'antecedente ROr c. 14, II, p. 470, dove si punisce un reato simile, e RTa c. 13.4, p. 32: sull'atteggiamento dei monaci verso la sessualità, spesso oggetto di grossolani fraintendimenti, cfr. G. PICASSO, Monaci e chierici di fronte alla sessualità, in Comportamenti e immaginario della sessualità nell'alto medioevo (Spoleto, 31 marzo - 5 aprile 2005), Spoleto 2006 (Settimane di studio del Centro italiano di studi sull'alto medioevo, 53), pp. 794-804 e 808-809; inoltre anche G. ARCHETTI, "Mensura victus constituere". Il cibo dei monaci tra Oriente ed Occidente, in L'alimentazione nell'alto medioevo: pratiche, simboli, ideologie (Spoleto, 9-14 aprile 2015), Spoleto 2016, (Settimane di studio del Centro Italiano di studi sull'alto medioevo, 53) pp. 780-792; ID., op. cit. (n. 18), pp. 563-578. Tutte le regole suppongono che il monaco rimproverato risponda prostrandosi a terra e chiedendo perdono fino a quando l'abate, o chi per lui, lo riterrà opportuno.

${ }^{50}$ Quest'ultima comporta, infatti, anche conseguenze sacramentali oltre alla separazione dalla comunità dei fedeli, che è invece l'unico effetto della scomunica monastica: cfr. S. PRICOCO (cur.), La regola di san Benedetto e le regole dei Padri, Milano 1995 (Scrittori greci e latini), p. 292. D’altra parte, ha osservato R. E. REYNOLDS, Rites of separation and reconcilation in the early middle ages, in Segni e riti nella Chiesa altomedievale occidentale (Spoleto, 11-17 aprile 1985), Spoleto 1987 (Settimane di studio del Centro italiano di studi sull'alto medioevo, 33), pp. 406-409, la scomunica venne molto presto articolandosi in varie forme, che per il mondo monastico sono state accuratamente esaminate da I. ROSÉ, Exclure dans un monde clos? L'excommunication dans les règles monastiques de l'antiquité tardive et du Haut Moyen Âge, in G. BÜHER-THIERRY - S. GIOANNI (cur.), Exclure de la communauté chrétienne. Sens et pratiques sociales de l'anathème et de l'excommunication (IVe-XIIe siècle), Turnhout 2015 (Collection haut Moyen Âge, 23), pp. 119-142.

${ }^{51} \mathrm{R} 4 \mathrm{P}$ 5.15.4-6, I, pp. 202-204: la scomunica è prevista per il vaniloquio e per risate vel scurrilitate sermonis, oltretutto senza neppure ammonizioni previe, un'assenza quest'ultima singolare, secondo A. DE VOGÜÉ, Règle des quatres Pères. Introduction, in ID. (ed.), Les règles des saints Pères, cit., I, p. 88, in un testo così ricco di citazioni scritturistiche.

${ }^{52}$ Quest'ultima motivazione riguardante l'espulsione è costantemente presente nelle regole. L'intera procedura in ROr cc. 32-35, II, pp. 488-49o, debitrice in parte dall'antecedente Secunda regula Patrum, benché le origine della ROr vadano probabilmente collocate a Condat, nella regione del Giura (cfr. A. DE VOGÜÉ, Règle orientale. Introduction, in ibid., II, pp. 435-454).

${ }_{53}$ Rispettivamente RMag c. 13.6o-61 et 66-67, II, pp. 44 e 46, e RB cc. 24 et 44.9-10, II, pp. 544 e 594. Sui tre richiami v. RMag c. 12.1-7, II, pp. 32-34, e RB c. 23.1-4, II, p. 542, che, a differenza del Maestro, prevede sia pubblico solo l'ultimo.

${ }^{54}$ Questa complessa procedura è descritta, con le sue specifiche penitenze, in RMag c. 13.1-49, 54-59, 62-65, et c. 14.1-78, II, pp. 34-46 e 48-6o; sul limite di tempo per l'emendazione v. RMag c. 13.68-73, II, pp. 46-48, ed è in relazione a esso che RMag c. 13.50-52, II, p. 42, aggiunge un digiuno di due giorni se il peccato fosse particolarmente grave, altrimenti un ritardo nel pasto con la somministrazione di acqua e di pane di mediocre qualità (riguardo alle differenti tipologie di quest'ultimo in uso nei monasteri cfr. G. ARCHETTI, Noli pane satiari. Il pane sulla tavola dei monaci, in ID. (ed.), op. cit. (n. 10), pp. 1690-1695); I. ROSÉ, op. cit., (n. 50), pp. 132-133, ha messo bene in chiaro la dimensione escatologica soggiacente alla cerimonia strutturata dal Maestro. ${ }_{55}$ Cfr. i puntuali rilievi di A. DE VOGÜÉ, La règle de saint Benoît. V: Commentaire historique et critique, Paris 1971 (Sch, 185), pp. 724-726, e di I. ROSÉ, op. cit., (n. 50), pp. 133-135.
} 
scomunicato $^{56}$, le molteplici iniziative che deve assumere prima di decretarne l'allontanamento dal cenobio ${ }^{57}$, infine la possibilità di riammetterlo - su sua richiesta e promessa di emendarsi pro quo egressus est - fino a tre volte, se RB c. 29 fosse da interpretarsi alla luce dei sei capitoli precedenti ${ }^{8}$. In tale caso sarebbe interessante rilevare un'altra divergenza tra le due regole: si è visto che per la RMag una seconda caduta appare irredimibile poiché, secondo il de Vogüé, essendo stata esemplata prima della diffusione della penitenza reiterabile irlandese - avvenuta verso la fine del secolo VI - essa conosce soltanto l'antica prassi dell'espiazione canonica59. Ciò però dovrebbe valere anche per la RB, tuttavia pure se la nostra ipotesi fosse corretta mi sembra che la riflessione dell'illustre studioso francese rimanga valida: solamente, si dovrebbe concludere per la presenza anche nel continente di pratiche penitenziali ripetibili non chiaramente formalizzate antecedenti l'arrivo degli usi insulari. Di esse, in effetti, alcune fonti fanno intravvedere le tracce e tra codeste si potrebbe perciò forse annoverare pure la $\mathrm{RB}$, anche perché è proprio la vita monastica a costituire il modello di riferimento di dette procedure ${ }^{60}$.

Segnalato en passant come la scomunica sia prevista solo per pochi reati nelle regole dell'area arelatense, prive di un vero e proprio codice penale ${ }^{61}$, il modello proposto dalle regole italiane è ripreso da quelle spagnole, secondo un orientamento in generale prossimo alla RB, ossia volto a un fine medicinale. Così la RIs e la RComp contempla- no varie iniziative antecedenti alla scomunica e la prima esclude in assoluto la cacciata del peccatore dal chiostro, pure se manifestasse gravissimi vizi comportanti ripetute scomuniche, ne forte [...] ore diaboli devoretur ${ }^{62}$. La stessa applicazione della sanzione prevede numerose fasi, minutamente descritte dalla RComp e dalla RComm, onde offrire all'impenitente plurime occasioni di riscatto prima di deciderne l'espulsione ${ }^{6_{3}}$. La RIs e la RComm riprendono dalla RMag la durata biduana o triduana della scomunica, ma la prima la prevede anche diuturna e in tale caso le collega un digiuno a pane e acqua ${ }^{64}$ : presente in precedenza nel solo Pacomio ${ }^{65}$, segnalare questa misura ci permette di svolgere due osservazioni conclusive.

Innanzi tutto, lo sviluppo nelle regole di un codice penale - al quale si affiancano costantemente norme repressive per reati isolati - ha permesso ai legislatori monastici di inserire nella scomunica, o di affiancare a essa, altre tipologie di punizione, pure previste dalle più antiche regole. Tra esse vi è appunto il digiuno, inteso come semplice ritardo nell'assunzione del pasto o come astinenza parziale o totale dal cibo, e minacciato in prevalenza per il mancato rispetto degli orari oppure in alternativa ad altre pene ${ }^{66}$. La RMag lo impiega largamente, mentre la RFe ne raccomanda la moderazione, contemplando delle interruzioni se dovesse essere protratto per tre giorni ${ }^{67}$. Altre sanzioni diffuse sono quelle corporali, perlopiù inflitte per peccati molto importanti ${ }^{68} \mathrm{e}$ soprattutto ai monaci giovani: dopo un accenno nell'Ordo

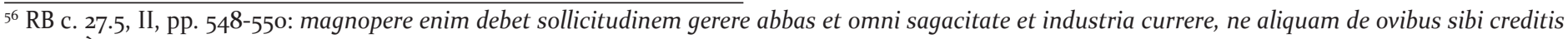
perdat. Ė, in effetti, specialmente in questa situazione che si esprime la gerarchia comunitaria fondata sull'autorità dell'abate, la quale, tuttavia, si declina sempre in termini di servizio pastorale finalizzato, appunto, alla salvezza delle anime dei monaci: cfr. a riguardo S. PACIOLLA, L'abate "vicario di Cristo": l'autorità nella vita monastica, in Angelicum, 85 (2008), pp. 934-941, e I. ROSÉ, op. cit., (n. 50), pp. 123-126 e 133-134. Per la procedura di scomunica e la sua soddisfazione - che in Benedetto, coerentemente, non prevede alcun limite di tempo - RB cc. 25-26 et 44.1-8, II, pp. 546-548 e 592.

${ }^{57}$ Benedetto ne tratta nei cc. 27 e 28, II, pp. 548-552, il secondo dei quali, a giudizio di A. DE VOGÜÉ, op. cit. (n. 55), pp. 737-741, riguarda non gli impenitenti ma i monaci ricaduti, dopo il perdono, in una nuova scomunica. Anche di costoro si cerca in tutti i modi il recupero e la loro stessa espulsione è inquadrata in prospettiva medicinale e non vendicativa, cfr. RB c. 28.6-8, II, p. 552 (il commento in ibid., pp. 740-741).

${ }^{8}$ Di tutt'altro avviso, in effetti, il de Vogüé (ibid., pp. 741-742, mi pare tuttavia con un'oscillazione alle pp. 724-725), per il quale questo capitolo deve essere collegato a RMag c. 64, II, p. 290, dove si tratta del monaco che exierit frequenter de monasterio e non dell'espulso. Mi permetterei però di rilevare, oltre alla posizione del passo nella RB, come in esso si aggiunga, rispetto al Maestro, la precisazione proprio vitio quale motivo per cui frater egreditur [...] de monasterio; inoltre in vari manoscritti della RB a egreditur si aggiunge aut proicitur o aut proiciatur (v. RB c. 29, II, p. 554, alla corrispondente n. di edizione). ${ }^{59}$ Cfr. RMag c. 14, II, p. 59, n. 68-69.

${ }^{60}$ Su queste pratiche cfr. G. PIANA, Peccati e penitenza nel medioevo, in ID. - G. PICASSO - G. MOTTA, A pane e acqua. Peccati e penitenze nel medioevo, Novara 1986, pp. 11-12, e M. G. MUZZARELLI, Penitenze nel medioevo. Uomini e modelli a confronto, Bologna 1994 (Il mondo medievale. Sezione di storia delle istituzioni della spiritualità e delle idee, 22), pp. 27-32.

${ }^{61}$ L'unica a dare indicazioni in proposito è RCae c. 23, p. 154

${ }^{62}$ RIs c. 16.2, col. $885 \mathrm{AB}$, mentre per le misure previe v. i cc. 14-16.2, coll. $884 \mathrm{~B}-885 \mathrm{~A}$, che comunque riguardano solo le colpe lievi perché per quelle gravi appare chiaramente sottinteso come il monaco non possa evitare la scomunica: un preciso elenco di entrambe le tipologie di peccato la RIs lo propone al c. 17 , coll. 885 B-886C. Per la RComp cfr. il c. 17, col. 1107B.

${ }^{63}$ Rispettivamente RComp cc. 15-16, coll. 1106B-1107 B - nel complesso quattro procedure, con norme sempre più severe in rapporto al tipo di peccato che la regola modella sull'esempio di Isidoro, tuttavia organizzandole in relazione alla pena e, perciò, in modo meno lineare rispetto alla sua fonte - e RComm c. 14, coll. ${ }_{1121} \mathrm{D}-1122 \mathrm{D}$. Un aspetto comune alle due regole è costituito dalle ripetute provocazioni cui il penitente è oggetto durante la scomunica, onde evidentemente saggiarne lo spirito di umiltà e la disponibilità piena all'obbedienza.

${ }_{4}^{64}$ RIs c. 18.2-3, col. 887AB, alimenti da assumere nella refectio vespertina, mentre se la scomunica è biduana excommunicato nihil alimenti praebendum est; invece la RComm c. 14, col. 1122A, gli assegna un medium [...] paximatium et non ad satietatem aquam.

${ }_{5}^{6}$ RPIu c.1, p. 64, previsto per il calunniatore che neghi di esserlo.

${ }^{66}$ Già in RPPr c. 32, p. 21, il ritardo alla mensa può essere espiato con un ritorno digiuno alla cella o con altra penitenza: il passo è ripreso dalla ROr c. 37, II, p. 490, e ha un parallelo in RBa int. 97, pp. 128-129. Nella RB c. 43.13-17, II, p. 590, è invece prevista la sola astinenza dal vino, come in RMag c. 11.109-110, II, p. 30, attinente però a reati compiuti nel dormitorio. Sempre per ritardi all'ora del pasto e in alternativa ad altre punizioni v. RIs c. 9.1, col. 878A, mentre RComp c. 15, col. 1106BC, prevede un digiuno biduano o triduano per alcuni gravi peccati di lingua.

${ }^{67}$ RFe c. 24.14-15, p. 137; anche Ferreolo utilizza come pena di preferenza il digiuno applicandolo a fattispecie molto differenti tra loro, sia perché la RMag è una delle sue fonti principali, sia perché la sua regola è una delle meno severe, preferendo in effetti incoraggiare il monaco piuttosto che punirlo in base al principio nec tam personas exigit damnare, quam culpas (c. 39.2, p. 145): su tutto ciò cfr. J. B. DE SALVERT, Règle de saint Ferreol. Introduction, in V. DESPREZ, op. cit. (n. 4), pp. 289-292.

${ }^{68}$ Per alcune esemplificazioni v. 3 RP cc. 9 et 13, II, pp. 538 e 540 (ebbrezza e furto: è l'unica regola 'lerinense' a prevedere questo tipo di punizione); RCae c. 5, p. 150 (menzogna); RFe c. 39.48-56, p. 148 (furto); RComp c. 15, col. 1106B (reati di lingua). 
monasterii agostiniano ${ }^{69}$ sono le due regole italiane a precisarne la somministrazione, secondo criteri tra loro differenti. La RMag, infatti, le sostituisce alla scomunica per i monaci sotto i 15 anni, poiché gli adulti iam intellegunt quomodo paeniteri et emendari debeant [...] quod mali committunt, ossia principalmente nella dimensione dell'interiorità, una consapevolezza ritenuta dal Maestro non ancora lucida nel giovane ${ }^{70}$. La RB, secondo sua abitudine, sviluppa tale riflessione e perciò le estende a tutti coloro che, per età o per condizione personale, non sono in grado di comprendere adeguatamente la grave portata della scomunica ${ }^{71}$. Quanto al numero dei colpi, solo due regole ne trattano: la regula Aureliani li fissa in 39, in ragione del loro scopo correttivo e non afflittivo, ma la RComm ne prevede ben 100 se un monaco e una monaca entrassero in confidenza tra loro, incontrandosi durante un viaggio effettuato per commissioni affidate loro dall'abate ${ }^{72}$. Si tratta di un problema caratteristico dei monasteri doppi, istituzione diffusa nell'area spagnola per la quale questa regola è stata scritta e che, presentando altre situazioni originali, estende l'impiego delle pene corporali a una casistica alquanto ampia73.
Questa complessa organizzazione della penitenza trova il suo ideale punto di approdo negli scritti di san Colombano, dai quali non a caso abbiamo preso le mosse. In effetti, le norme finora esaminate erano dirette unicamente ai monaci, valendo per il resto dei fedeli, come sopra si èaccennato, l'antica penitenza canonica. Essa si svolgeva in forma pubblica, era amministrata dal vescovo ed effettuabile una sola volta nella vita, infine lasciava al peccatore, dopo l'assoluzione, una serie di pesanti e perpetue interdizioni ${ }^{74}$. Viceversa, saranno proprio la RCe e il penitenziale colombaniano a diffondere in tutto l'Occidente il sistema della penitenza privata insulare - di cui solamente le regole spagnole sono un riflesso ${ }^{75}$ - estendendola inoltre al di fuori dell'ambiente claustrale, determinando così una prassi espiatoria destinata a protrarsi, con modifiche, oscillazioni e integrazioni, fino alla riforma del Lateranense $I^{76}$. A connotare la Rce, infatti, è il sobrio spazio riservato alla scomunica eall'espulsione ${ }^{77}$ mentre larga attenzione è dedicata alla casistica, puntualissima nel collegare ciascun peccato a una specifica penitenza ma altrettanto spesso affastellando le norme con un certo disordine, aspetti entrambi caratteristici dei libri paenitentiales. Senza smar-

\footnotetext{
${ }_{69}$ AUGUSTINUS, Ordo monasterii c. 10, in P. VERHEJEN (ed.), La règle de saint Augustin, I. Tradition manuscrite, Paris 1967 (Études augustiniennes, 29), p. 152, per chi ostinatamente violi le norme: l'espressione talis fuerat aetas ipsius porta a supporre che la punizione riguardi, appunto, i monaci giovani. La misura è implicitamente ripresa da RTa c. 13.9, p. 33 .

$7^{70}$ RMag c. 14.79-86, II, pp. 6o-62, e ipotizzerei anche perché si riteneva all'epoca che nei giovani gli istinti corporei assumessero un ruolo alquanto rilevante, perciò punire il corpo sarebbe risultato particolarmente efficace: lo conferma, a mio giudizio, la ripresa della norma in RIs c. 18.2, col. 887A, con la motivazione ut quos aetatis infirmitas a culpa non revocat, flagelli disciplina compescat.

${ }^{71} \mathrm{RB}$ c. 23 , II, p. 542: si intellegit qualis poena sit, excommunicationi subiaceat; sin autem improbus sit, vindictae corporali subdatur, cfr. in proposito A. DE VOGÜÉ, op. cit. (n. 55), pp. 742-745, anche per un confronto con la RMag. Principio decisivo, dunque, è l'effettiva autocoscienza della colpa e della necessità di espiarla, un criterio, secondo J. HILLNER, Monks and children: corporal punishment in late antiquity, in European review of history, 16 (2009), pp. 773-791, soprattutto pp. 780-783, di origini scritturistiche e patristiche ma altresì classico-pagane, come proverebbe anche la somministrazione della pena non col flagello ma mediante l'impiego della ferula, secondo l'uso scolastico romano: sul tema cfr. anche G. ARCHETTI, Ildemaro a Brescia e la pedagogia monastica nel commento alla Regola, in ID. - A. BARONIO (cur.), San Faustino Maggiore di Brescia il monastero della città, Atti della giornata nazionale di studio (Brescia, Università Cattolica del Sacro Cuore, 11 febbraio 20015), Brescia 2006 [= Brixia sacra, s. III, 11/1 (2006)], pp. 158-162; G. ARCHETTI, L'educazione dei «pueri oblati»: reclutamento, formazione e finalità, in M. E. VARELA-RODRÍGUEZ (cur.), La historiografia medieval davant la crisi. La historiografía medieval ante la crisis, Girona 2015 (Estudis de Cultura Escrita i Visual, 3), pp. 67-124; ID., op. cit. (n. 18), pp. 527-582. Unica eccezione al criterio mi pare prevista dal c. 71, II, p. 668, che prevede la corporalis vindicta, senza eccezioni e senza scomunica, per chi non intende emendarsi dal rifiuto di obbedienza (e per gli ostinati irredimibili è sancita l'espulsione).

${ }_{72}$ Rispettivamente Regula Aureliani ad monachos cc. 41 et 42, PL 68, col. 392AB, e RComm c. 15, col. 1123 C: nel secondo caso si parla di un semplice scambio di parole!

${ }^{73} \mathrm{Ad}$ es. pene corporali sono determinate per i figli di coloro che entravano in un monastero 'familiare' - RComm c. 6, col. 1116AB - o per chi si faceva monaco in età anziana - RComm c. 8, col. $1117 \mathrm{AB}$ - o infine per i piccoli pecorai - RComm c. 9, col. 1117D. Su tali peculiarità v. sopra, n. 19.

${ }^{74}$ Soprattutto codeste ultime due caratteristiche ne avevano provocato un'irreversibile crisi nei secoli V-VI: cfr. G. PIANA, op. cit. (n. 6o), pp. 8-10; M. G. MUZZARELLI, op. cit. (n. 6o), pp. 13-18 e 23-25; G. MOTTA, Il tempo della penitenza, in G. ARCHETTI - A. BARONIO (cur.), Tempus mundi umbra aevi. Tempo e cultura del tempo tra medioevo ed età moderna, Atti dell'incontro nazionale di studio (Brescia, 29-30 marzo 2007), Brescia 2008 (Storia, cultura e società, 1), pp. 187-190.

${ }_{75}$ Lo si nota, in particolare, per l'impiego della carcerazione nei confronti dello scomunicato, prevista da RCe c. 6, p. 5o, fino a che non dia espliciti segnali di resipiscenza: la misura, assente nella RIs, è prevista con varie aggravanti sia nei cc. 15-16 (col. 1107AB) della RComp, sia nella RComm c. 14, coll. 1121D-1122D. Secondo I. ROSÉ, op. cit., (n. 50), pp. 121-123, 128-129, e 130-132, l'introduzione del carcere nella scomunica modificò il significato di quest'ultima rispetto alla tradizione precedente, da una separazione cioè fisica e spaziale a un isolamento sociale che ne accentuò i tratti penitenziali, e ciò potrebbe altresì spiegare perché, in quel periodo, iniziasse a diffondersi la prassi, da parte di vescovi e concili, di condannare i laici rei di gravi peccati alla reclusione temporanea in monastero: cfr. in proposito J. HILLNER, L'enfermement monastique au VI e siècle, in I. HEULLANT-DONAT - J. CLAUSTRE - É. LUSSET (cur.), Enfermements: le cloître et la prison (VI ${ }^{e}-X V I I I^{e}$ siècle), Actes du colloque international (Troyes-Bar-sur-Aube-Clairvaux, 22-24 octobre 2009), Paris 2011 (Homme et société, 38), pp. 39-56.

${ }^{76}$ T. M. CHARLES-EDWARDS, The penitential of Columbanus, in M. LAPIDGE (cur.), op. cit. (n. 1), pp. 218-219 e 238, ha messo in luce come il penitenziale di Colombano raccolga, da un lato, la tradizione irlandese precedente, per la quale ogni singolo penitenziale si indirizzava o ai monaci, o al clero, o ai laici, dall'altro lato avvii la prassi successiva rivolgendosi con il suo scritto a tutti e tre, pur mantenendo al proposito sezioni distinte. Immensa è la bibliografia attorno alla penitenza tariffata e ai libri penitenziali: mi limito a segnalare, oltre ai fondamentali lavori di C. VOGEL, Les libri paenitentiales, Turnhout 1978 (Typologie des sources du Moyen Âge occidental, 27), specialmente pp. 34-43 - con la Mise à jour di A. J. FRANTZEN, Turnhout 1985 - e Il peccatore e la penitenza nel medioevo, Torino 1988, pp. 15-20, G. PIANA, op. cit. (n. 6o), pp. 13-14 e 18-24; M. G. MUZZARELLI, op. cit. (n. 60), pp. 34-41; G. MOTTA, op. cit. (n. 39), pp. 55-75; M. DUNN, Paradigms of penance, in The journal of medieval monastic studies, 1 (2012), pp. 17-39. In particolare per la diffusione dei penitenziali irlandesi nel continente R. KOTTJE, Überlieferung und rezeption der irischen Bußbücher auf dem Kontinent, in H. LÖWE (cur.), op. cit. (n. 36), pp. 511-523.

77 La RCe non presenta una precisa procedura per la scomunica trattandone solo nel c. 6, (v. sopra, n. 75). Quanto all'espulsione, è citata solo in RCe c. 15, p. 166 - uno dei testi aggiunti allo scritto originario, cfr. T. M. CHARLES-EDWARDS, op. cit. (n. 76), pp. 232-237 - per chi suum praepositum dispexerit aut regulam blasphemaverit, a meno che non si penta e si umili pubblicamente.
} 
rirci troppo nei dettagli, dopo avere ricordato la necessità di una piena e completa confessione delle colpe e avere perciò, con coerenza, introdotto una saemipaenitentia per chi si riconosce colpevole di quelle $\operatorname{lievi}^{78}$, nei primi cinque capitoli la Rce individua nelle punizioni corporali il mezzo prevalente di emendazione ${ }^{79}$. Nel cap. 4, tuttavia, è introdotta pure la superpositio $^{80}$, sovente estesa a tre giorni, la quale diventa la forma principale di penitenza fino all'inizio della seconda parte, dove il suo posto è preso dal digiuno a pane e acqua. Codesto è già inflitto, nel cap. 8 , a chi ad praepositum audeat dicere: 'non tu iudicabis causam meam': prolungato anche per due o tre giorni e affiancato, nella RCe, dalla recita dei salmi e dai verbera, diverrà il simbolo stesso della penitenza tariffata, al punto che le verbe paenitere qui revient inlassablement dans nos textes ne signifie pas autre que ieiunare ${ }^{81}$. In alcuni capitoli, infine, è presente il principio della commutazione, altro aspetto peculiare della procedura irlandese talvolta sbrigativamente indicato quale principale causa del suo abbandono, avendo favorito l'arbitrarietà della penitenza e il suo scivolamento nel lassismo così fortemente deplorati dai riformatori carolingi ${ }^{82}$. In realtà, le tariffe di commutazione sono già presenti nei primi libri penitenziali per l'evidente necessità di rendere effettuale la penitenza imposta dal confessore: costui, infatti, assegnava al peccatore, per ciascuna colpa da lui riconosciuta, una pena consistente in vari anni di digiuno a pane e acqua, talché facilmente si raggiungeva, anche nell'ambito di un unico atto sacramentale, un periodo di espiazione spesso eccedente la prevedibile durata della vita del reo. La commutazione consentiva pertanto di trasformare questa 'penitenza teorica' in una prassi reale affiancando al digiuno altre misure compensative, dunque il lassismo fu dovuto al modo in cui queste tariffe compensatorie furono, in progresso di tempo, interpretate e non alla loro specifica natura ${ }^{83}$.

Non è questo oltretutto il caso di Colombano, le cui commutazioni non sono punto leggere, benché senza dubbio contraddittorie con altre norme della $\mathrm{RCe}^{84}$ : in effetti, l'ultimo esito del percorso che si è cercato di tracciare è l'anonima RcP, dipendente dall'opera dell'abate insulare ${ }^{85}$. Lo scritto è strutturato come un vero e proprio penitenziale e, aldilà della casistica specifica, si segnala per l'estrema severità: organizzando i reati in un preciso codice penale, la RcP li punisce quasi tutti con la scomunica - la quale prevede sempre la reclusione del peccatore - e con l'espulsione in assenza di rapida resipiscenza ${ }^{86}$, laddove si è visto nella tradizione antecedente prevalere, in genere, un orientamento volto al recupero. È codesta una delle ragioni che spiegano il successo finale della RB rispetto al modello monastico irlandese, ancor prima della sanzione ufficiale voluta da Ludovico il $\mathrm{Pio}^{87}$.

\footnotetext{
${ }_{78}$ Rispettivamente RCe c. 1, p. 144 - Colombano indica per la confessione i momenti antecedenti al pasto o al coricarsi a letto, ma aggiunge vel quandocumque fuerit facile - e c. 3, p. 148, con la clausola limitativa ai peccati non comportanti la superpositio.

${ }^{79}$ L'espressione utilizzata è percussionibus, secondo l'uso irlandese, variabili in rapporto al reato, tuttavia nel c. 9, p. 154, si limitano a 25 il massimo di colpi che possono essere inflitti per le colpe lievi. Nella seconda parte, che inizia col c. 10, questo tipo di punizione è indicato con la parola verbera.

${ }^{80}$ Nella RCe indicherebbe la privazione della parola e talvolta del nutrimento - cfr. A. DE VOGÜÉ, op. cit. (n. 24), p. 76 - ma nella prassi penitenziale tariffata successiva implicava, secondo C. VOGEL, Les 'libri paenitentiales' (cit. in n. 76), pp. 37-38, il prolungamento del digiuno per tre ore rispetto al momento del pasto $\mathrm{o}$, più frequentemente, un'astinenza completa dal cibo per due dì e una notte, ma talvolta anche per tre o quattro giorni.

${ }^{81}$ C. VOGEL, ibid., p. 37.

${ }^{82}$ Repudiatis ac penitus eliminatis libellis quos penitentiales vocant, quorum sunt certi errores, incerti auctores in concilium Cabillonense a. 813 c. 38 , in A. Wermingoff (ed.), Concilia aevi Karolini, Hannoverae et Lipsiae 1906 (MGH, Concilia, 2/1), p. 281: a questo giudizio si accompagnò un tentativo di riforma che, in realtà, approdò a un duplice regime penitenziale, cfr. C. VOGEL, ibid., pp. 39-42; G. PICASSO, op. cit. (n. 39), pp. 135-136; G. MOTTA, op. cit. (n. 39), pp. 67-72; A. H. GAASTRA, Penitentials and canonical authority, in R. CORRADINI - R. MEENS - C. POSSEL - P. SHAW (cur.), Texts and identities in the early middle ages, Wien 2006 (Forshungen zur Geschichte des Mittelalters, 12), pp. 191-203.

${ }^{83}$ Decisive osservazioni in proposito da parte di C. VOGEL, ibid., pp. 38-39 e 47-54, ma v. anche pp. 54-58 per gli ulteriori motivi che causarono la crisi del sistema penitenziale tariffato.

${ }^{8} \mathrm{Ad}$ es. RCe c. 15, p. 164, punisce chi solus cum sola femina sine certis personis familiariter loquitur con la superpositio o con due dì a pane e acqua o con 200 colpi, tuttavia in RCe c. 4, p. 148, la superpositio era considerata equivalente a 50 colpi. In generale, tutto il sistema di sanzioni della RCe pare confermarne il carattere stratificato, v. in proposito A. GRANATA, Regula coenobialis, in ID. - I. BIFFI (cur.), San Colombano. Le opere, Milano 2001 (Di fronte e attraverso, 555), pp. 269-274.

${ }_{5}$ Cfr. F. VILLEGAS, La "Regula cuiusdam Patris", cit., pp. 3 e 135-140, e J. LAPORTE, s.v., Regula cuiusdam Patris ad monachos, in Dizionario degli Istituti di Perfezione, VII, Roma 1983, coll. 1571-1573, che ha altresì ben lumeggiato le novità di questo scritto rispetto alle regole dell'abate irlandese

${ }^{86}$ Tale misura, in effetti, appare quasi ordinaria e talvolta non è nemmeno preceduta dalla scomunica, ad es. in RcP c. 16.3-4, pp. 23-24, contro colui che rispondesse cum clamore et acerbiore sermone e non si correggesse immediatamente dopo essere stato ripreso.

${ }^{87}$ Gli stessi monasteri colombaniani accoglieranno la RB perché, ha osservato A. DE VOGÜÉ, op. cit. (n. 24), pp. 32-33, gli scritti del santo irlandese erano carenti rispetto alla strutturazione della comunità, ma ciò avverrà tanto più agevolmente per il comune riferimento delle due tradizioni a Cassiano. Su tempi e contesti di tale recezione cfr. T. M. CHARLES-EDWARDS, op. cit. (n. 36), pp. 383-388.
} 